\title{
Social modulation of learning in rats
}

\author{
Ewelina Knapska, ${ }^{1,2,3}$ Marta Mikosz, ${ }^{2}$ Tomasz Werka, ${ }^{2}$ and Stephen Maren ${ }^{1}$ \\ ${ }^{1}$ Department of Psychology, Neuroscience Program, University of Michigan, Ann Arbor, Michigan 48109, USA; ${ }^{2}$ Nencki Institute of \\ Experimental Biology, Poland, 02-093 Warszawa, Poland
}

\begin{abstract}
It is well known that emotions participate in the regulation of social behaviors and that the emotion displayed by a conspecific influences the behavior of other animals. In its simplest form, empathy can be characterized as the capacity to be affected by and/or share the emotional state of another. However, to date, relatively little is known about the mechanisms by which animals that are not in direct danger share emotions. In the present study, we used a model of between-subject transfer of fear to characterize the social interaction during which fear is transmitted, as well as the behavioral effects of socially transmitted fear. We found that (1) during social interaction with a recently fearconditioned partner, observers and demonstrators exhibit social exploratory behaviors rather than aggressive behaviors; (2) learning and memory in a shock-motivated shuttle avoidance task are facilitated in rats that underwent a social interaction with a partner that had been fear conditioned; and (3) a brief social interaction with a recently fearconditioned partner immediately before fear conditioning increases conditioned freezing measured on the next day. The observed effects were not due to a stress-induced increase in pain sensitivity or analgesia. Collectively, these data suggest that a brief social interaction with a cage mate that has undergone an aversive learning experience promotes aversive learning in an otherwise naïve animal. We argue that socially transferred fear is an adaptation that promotes defensive behavior to potentially dangerous situations in the environment.
\end{abstract}

Human empathy can be defined as the ability to experience and share the thoughts and feelings of others (de Waal 2008). Obviously, this is a complex social phenomenon that, until recently, has received much attention from philosophers and psychologists rather than neuroscientists (Decety and Lamm 2006). However, in its simplest form, empathy can be characterized as the capacity to be affected by and/or share the emotional state of another (de Waal 2008). Tuning one's emotional state to that of another increases the probability of similar behavior, which thereby allows rapid adaptation to environmental challenges (Hatfield et al. 1994). This social adaptation may be particularly important for emotions that signal a potential danger, such as fear. Although one can learn about potentially harmful stimuli by directly experiencing an aversive event, observation or interaction with a conspecific in danger and/or in pain may also provide information about threats in the environment. There is a vast literature on learning about direct danger (Maren 2001) as well as sharing emotions through observation (see, e.g., Church 1959; Langford et al. 2006; Olsson and Phelps 2007). However, relatively little is known about the mechanisms by which animals that are not in a direct danger share emotions.

We have recently designed an experimental rat model of between-subject transfer of emotional information (Knapska et al. 2006). In this model, rats are housed in pairs and one member of the pair (the "demonstrator") is removed and subjected to fear conditioning. After the fear-conditioning episode, the conditioned animal is allowed to interact with its naïve cage mate (the "observer"). We showed that the demonstrator's fear is socially transferred to the observer, resulting in both rapid increase in exploratory behavior of the observer and a pattern of c-Fos activation in the observer's amygdala that parallels that of the shocked demonstrators.

\footnotetext{
${ }^{3}$ Corresponding author.
}

E-mail e.knapska@nencki.gov.pl; fax 48-22-8225342.

Article is online at http://www.learnmem.org/cgi/doi/10.1101/lm.1670910.
These results suggest that the social interaction between the demonstrator and observer results in a transfer of information that promotes aversively motivated learning in the observer. However, the nature of the social interaction and how it comes to influence aversively motivated behavior is not known. Therefore, the present study aimed to characterize the behavior of both the demonstrators and observers during their social interaction and further characterize the nature of the influence of socially transmitted fear on aversively motivated learning and memory. We hypothesized that the social interaction between observers and demonstrators would result in a social transfer of fear that would promote learning and memory of both active defensive responses (avoidance) as well as defensive immobility (freezing). To test this hypothesis, we carried out five experiments that examined the nature of the social interaction between shocked demonstrators and observers (Experiment 1), the acquisition and retention of active avoidance (Experiment 2), and conditioned freezing (Experiment 3). Because it is not clear if social transfer effects can be observed among unfamiliar animals and to control for social buffering, we also compared the social transfer of fear in familiar and unfamiliar rats (Experiment 4). To control for the possible influence of different pain sensitivity thresholds in the observers paired with shocked demonstrators, we carried out pain tests (Experiment 5).

\section{Results}

Sniffing and allogrooming are increased in observers during social interaction with a fear-conditioned demonstrator (Experiment 1)

In order to characterize behavior of demonstrators and observers during their interaction, we analyzed the number and/or duration of the nonsocial behaviors-(1) rearing, (2) self-grooming; social exploratory behaviors-(3) sniffing partner in the anogenital area, (4) sniffing partner in the head area, (5) allogrooming (grooming of the partner); and agonistic behaviors-(6) threat posturing, (7) leaping, (8) boxing, (9) wrestling, and (10) biting. 
All the abovementioned behaviors were analyzed in four groups of animals: the fear-conditioned demonstrators ( $\mathrm{Sd}, n=8$ ), the observers paired with fear-conditioned demonstrators (So, $n=8$ ); the demonstrators merely exposed to the novel cage (NSd, $n=7$ ), and the observers paired with non-shocked demonstrators (NSo, $n=$ 7). For a detailed description of the experimental groups, see Table 1.

None of the agonistic behaviors were observed. The results for the other behaviors are summarized in Table 2. The demonstrators from the $S$ group reared more often than the observers from both the $\mathrm{S}$ and NS groups. On the other hand, all observers showed significantly longer sniffing in their partner's anogenital areas. Moreover, the observers from the $S$ group had significantly increased allogrooming. The data were analyzed with two-way ANOVAs $(\mathrm{d} / \mathrm{o} \times \mathrm{S} / \mathrm{NS})$, followed by Fisher's PLSD tests. There were the effects of d/o for the number of rearings: $F_{(1,26)}=7.55, P<0.05$ (Sd vs. So, $P<0.01$; Sd vs. NSo, $P<0.01$ ) and duration of sniffing in the anogenital area: $F_{(1,26)}=12.48, P<0.01$ (So vs. $\mathrm{Sd}, P<0.05$; So vs. NSd, $P<0.05$; NSo vs. Sd, $P<0.05$; NSo vs. NSd, $P<0.01$ ), as well as the effect of S/NS for the duration of allogrooming: $F_{(1,26)}=6.41, P<0.05$ (So vs. NSo, $P<0.05$; So vs. NSd, $P<$ $0.05)$. These results indicate that during the interaction, the observers were involved mainly in social exploratory behaviors; these behaviors were directed at their partners, and they aim at gaining information.

\section{Interaction with a recently fear-conditioned demonstrator enhances acquisition and retention of two-way avoidance response (Experiment 2)}

In order to determine whether acquisition of an active avoidance response is modulated by previous interaction with a fear-conditioned partner, we compared different parameters of learning in the observers paired with either a fear-conditioned demonstrator (So, $n=8$ ) or an animal merely exposed to the experimental cage (NSo, $n=8$ ) (see Fig. 1A). We also examined retention of the twoway avoidance response in observers trained after interacting with shocked or non-shocked demonstrators. Moreover, we compared the results of the animals housed in pairs and interacting with their partners with those obtained by rats that were single-housed and did not interact with other animals at any point of the experiment (SINGLE, $n=8$ ). In the first session that immediately followed the social interaction, we observed an increased number of avoidance responses and shorter latencies of these responses in the observers paired with fear-conditioned demonstrators (So group) in comparison to the observers paired with non-shocked demonstrators (NSo group). The significantly shorter latencies of instrumental responses were also seen during the second (retention) session. The SINGLE rats acquired the two-way avoidance response more slowly as was shown with the number of avoidance responses and response latencies. Retention of the

Table 1. Description of the experimental groups

\begin{tabular}{|c|c|c|}
\hline Abbreviation & Group & Description \\
\hline Sd & Shocked demonstrators & $\begin{array}{l}\text { Before social interaction the rats were subjected to } \\
\text { Pavlovian contextual fear conditioning. }\end{array}$ \\
\hline So & Shocked observers & $\begin{array}{l}\text { The rats were paired with shocked demonstrators } \\
\text { during social interaction. }\end{array}$ \\
\hline NSd & $\begin{array}{l}\text { Non-Shocked } \\
\text { demonstrators }\end{array}$ & $\begin{array}{l}\text { Before social interaction the rats were exposed to the } \\
\text { experimental cage without any training. }\end{array}$ \\
\hline NSo & Non-Shocked observers & $\begin{array}{l}\text { The rats were paired with non-shocked demonstrators } \\
\text { during social interaction. }\end{array}$ \\
\hline SINGLE & Single & $\begin{array}{l}\text { Single-housed rats were trained in fear conditioning } \\
\text { and two-way avoidance paradigms that did not interact } \\
\text { with another rat. }\end{array}$ \\
\hline
\end{tabular}

avoidance response measured on the next day was also significantly worse than in the animals that interacted with their partners before the training (Fig. 1B,C).

The number of avoidance responses was analyzed in five 10trial blocks with a three-way ANOVA (group $\times$ session $\times$ blocks) that showed the effects of group $\left(F_{(2,21)}=6.53, P<0.01\right)$, session $\left(F_{(1,21)}=26.57, P<0.001\right)$, and block $\left(F_{(4,84)}=18.28, P<0.001\right)$. Then the results were analyzed with two-way ANOVAs (group $\times$ blocks) for the first and second sessions, followed by Duncan post-hoc tests. For the first session there were the effects of group $\left(F_{(2,21)}=7.93, P<0.01\right.$; So vs. NSo, $P<0.05$; So vs. SINGLE, $\left.P<0.01\right)$ and block $\left(F_{(4,84)}=12.05, P<0.001\right)$. For the second session there were the effects of group $\left(F_{(2,21)}=4.31, P<0.05\right.$; So vs. SINGLE, $P<$ 0.05 ; NSo vs. SINGLE, $P<0.05)$ and block $\left(F_{(4,84)}=8.95, P<0.001\right)$. The latencies of the avoidance responses were significantly shorter in the So group in both the first and the second sessions compared to the NSo group. Moreover, the SINGLE rats had longer latencies in both the first and second sessions compared to the animals from the So and NSo groups (Kolmogorov-Smirnov two-sample tests) (for detailed statistical analysis, see Table 3). These results show that the observers paired with the fear-conditioned demonstrators better acquired and remembered the avoidance response than their partners that were paired with animals merely exposed to the experimental cage. Moreover, the performance of the observers paired with either shocked or non-shocked demonstrators before the training was better than the performance of rats that did not interact with other animals.

Because our previous results suggested that the observers from the $S$ group are more active (see Knapska et al. 2006), we also analyzed the frequency of crossings between the compartments during the period of adaptation (a), and the frequency of intertrial responses (ITRs) observed during intertrial intervals (ITIs) when neither conditioned stimulus (CS) nor unconditioned stimulus (US) was available. Indeed, we observed significantly increased frequency of crossings and ITRs in the So group comparing to the NSo and SINGLE groups during the first session of the twoway avoidance response training (see Fig. 1D). A two-way ANOVA (group $\times$ session) showed the effects of group $\left(F_{(2,21)}=\right.$ 16.40, $P<0.001)$ and session $\left(F_{(1,21)}=22.21, P<0.001\right)$, as well as interaction between group and session $\left(F_{(2,21)}=14.05, P<\right.$ 0.001 ) for the response rate that was observed during the adaptation period (before the first CS and US were applied). Then, the data were analyzed with two one-way ANOVAs for two sessions, followed by Duncan post-hoc tests that showed the significant effect for the first session $\left(F_{(2,21)}=26.55, P<0.001\right.$; So vs. NSo, $P<0.001$; So vs. SINGLE, $P<0.001)$. For the frequency of ITRs, a three-way ANOVA (group $\times$ session $\times$ blocks) revealed the effect of group $\left(F_{(2,21)}=8.43, P<0.01\right)$, as well as interactions between group and session $\left(F_{(2,21)}=5.32, P<0.05\right)$; session and block $\left(F_{(4,84)}=7.13, P<0.001\right)$; and group, session, and block $\left(F_{(8,84)}=3.36, P<0.01\right)$. Then the data were analyzed with two-way ANOVAs (group $\times$ blocks) for the first and second sessions, followed by Duncan post-hoc tests. For the first session, two-way ANOVAs showed the effect of group $\left(F_{(2,21)}=19.42, P<0.001\right.$; So vs. NSo, $P<0.001$; So vs. SINGLE, $P<0.001)$, as well as interaction between group and blocks $\left(F_{(8,84)}=2.14, P<0.05\right)$, whereas for the second session, two-way ANOVA (group $\times$ blocks) showed only the effect of blocks $\left(F_{(4,84)}=6.36, P<0.001\right)$. The obtained data indicate that the observers paired with fear-conditioned demonstrators were more active at the beginning of 
Table 2. Summary of mean numbers or durations of the behaviors observed during 10-min social interaction $( \pm$ SEM)

\begin{tabular}{lccccc}
\hline Group & $\begin{array}{c}\text { Rearing } \\
\text { (no) }\end{array}$ & $\begin{array}{c}\text { Self-grooming } \\
\text { (dur) }\end{array}$ & $\begin{array}{c}\text { Sniffing-anogenital } \\
\text { area (dur) }\end{array}$ & $\begin{array}{c}\text { Sniffing-head } \\
\text { area (dur) }\end{array}$ & $\begin{array}{c}\text { Allogrooming } \\
\text { (dur) }\end{array}$ \\
\hline Sd & $19.6 \pm 3.1$ & $58.5 \pm 14.7$ & $5.9 \pm 1.4$ & $4.0 \pm 1.3$ & $8.1 \pm 3.5$ \\
So & $8.8 \pm 2.1^{\mathrm{a}}$ & $98.0 \pm 34.0$ & $17.7 \pm 4.5^{\mathrm{b}, \mathrm{c}}$ & $3.8 \pm 1.2$ & $17.6 \pm 6.8^{\mathrm{e}, \mathrm{c}}$ \\
NSd & $12.3 \pm 2.0$ & $52.9 \pm 33.5$ & $2.9 \pm 1.1$ & $1.1 \pm 0.6$ & $2.7 \pm 2.4$ \\
NSo & $9.1 \pm 2.8^{\mathrm{a}}$ & $39.6 \pm 19.1$ & $19.0 \pm 6.4^{\mathrm{b}, \mathrm{d}}$ & $2.8 \pm 1.0$ & $1.2 \pm 1.2$ \\
\hline
\end{tabular}

(no) Mean numbers; (dur) durations (in seconds).

${ }^{a} P<0.01 ;{ }^{b} P<0.05$ vs. Sd.

${ }^{c} p<0.05 ;{ }^{d} p<0.01$ vs. NSd.

${ }^{\mathrm{e}} P<0.05$ vs. NSo.

the training than the observers paired with nonconditioned demonstrators and than single rats.

\section{Interaction with a recently fear-conditioned demonstrator enhances conditioned freezing (Experiment 3)}

To determine whether the facilitated acquisition of avoidance conditioning is related to the transfer of fear or simply due to enhanced locomotor activity in observers, we next examined the acquisition and retention of a conditioned freezing response characterized by behavioral immobility. We measured conditioned freezing of observers that interacted with a fear-conditioned demonstrator before being fear-conditioned themselves (So, $n=13$ ). We compared their results with the level of freezing in the animals that interacted with a nonshocked partner (NSo, $n=12$ ) and animals that did not interact at all (SINGLE, $n=8$ ) (see Fig. 2A). The level of postshock freezing during the TRAIN day did not significantly differ between groups (So: $52.37 \% \pm 6.95$; NSo: $35.78 \% \pm 8.18$; SINGLE: $41.34 \% \pm 10.72$ ). During the TEST day, however, we observed increased level of freezing in the animals from the So group at the beginning of the exposure to the fear-conditioned context in comparison to the animals from the NSo group. The level of freezing in the So and SINGLE animals was similar; see Figure $2 \mathrm{~B}$. A percentage of freezing in the subsequent minutes of training and testing was analyzed with two-way ANOVA (group $\times$ blocks) that showed the effect of blocks $\left(F_{(5,150)}=27.78, P<0.001\right)$ and the interaction between group and blocks $\left(F_{(10,150)}=2.21, P<0.05\right)$. Then, the data were analyzed with six one-way ANOVAs followed by Fisher's PLSD tests. There was a significant difference in the first minute of testing: $F_{(2,30)}=4.19, P<0.05$ (So vs. NSo, $P<0.05$; SINGLE vs. NSo, $P<0.05$ ). These results indicate that, similarly as in the avoidance training, acquisition and retention of a conditioned freezing response were better in the observers paired with the fear-conditioned demonstrators than in the animals paired with partners merely exposed to the experimental cage. 0.001 .
Interaction with a recently fear-conditioned demonstrator enhances conditioned freezing in unfamiliar observers

(Experiment 4)

To determine whether the effect of socially transferred fear occurs among unfamiliar animals, we tested the rats that were single-housed and had contact with one another only during the 10-min interaction preceding the fear-conditioning session (So, $n=12$; NSo, $n=12$ ). On the TEST day, we observed an increased level of freezing in the animals that interacted with fear-conditioned partners on the training day (see Fig. 3). A percentage of freezing in the subsequent minutes of training and testing was analyzed with two-way ANOVA (group $\times$ blocks) that showed the effects of group $\left(F_{(1,22)}=4.87, P<0.05\right)$ and blocks $\left(F_{(5,110)}=18.43, P<0.001\right)$. Then, the data were analyzed with six one-way ANOVAs that revealed the significant difference in the second minute of testing during TEST day $\left(F_{(1,22)}=4.96, P<0.05\right)$. The data indicate that acquisition and retention of a conditioned freezing response were faster in the observers paired with the fear-conditioned demonstrators than in the animals paired with the rats merely exposed to the experimental cage also among unfamiliar rats.
A.

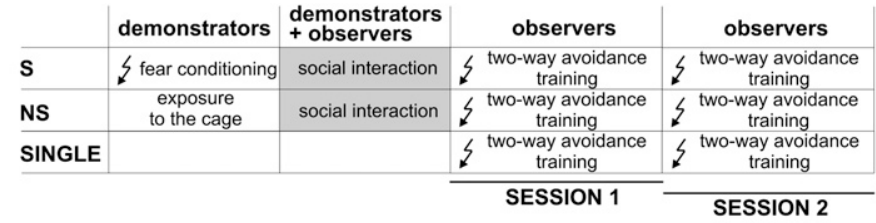

B.

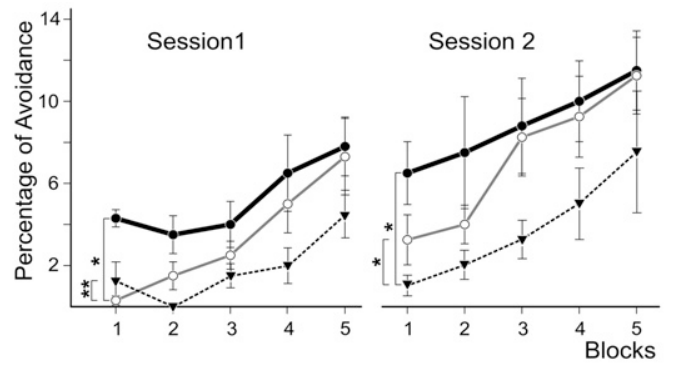

D.
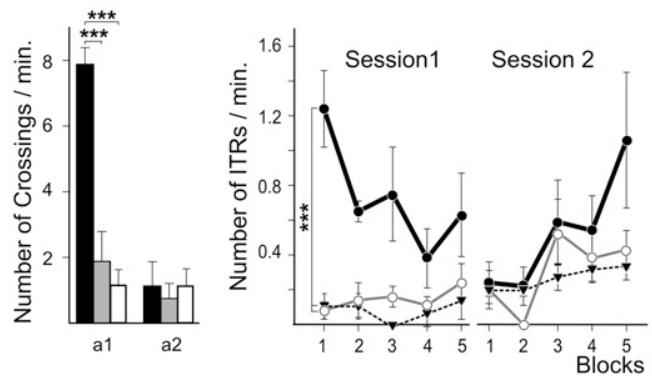

C.

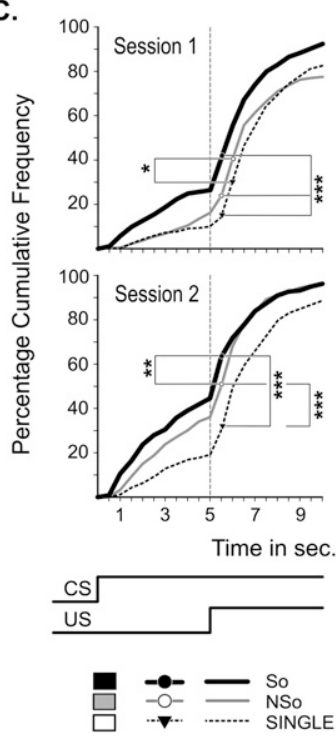

Figure 1. Interaction with a recently fear-conditioned partner improves acquisition and retention of two-way avoidance response. All behavioral parameters presented were registered during two consecutive 50-trial sessions of two-way avoidance training. (A) Scheme of the experiment. $(B)$ Percentage of avoidance response in five 10-trial blocks. (C) Cumulated distribution of latency of avoidance (shorter than $5 \mathrm{sec}$ ) and escape responses (longer than $5 \mathrm{sec}$ ). (D) Frequency of crossings during the adaptation period (before the first CS and US were delivered) in the first (a1) and the second (a2) session and during intertrial intervals (ITRs) in five 10-trial blocks. (So) Observers paired with fearconditioned demonstrators; (NSo) observers paired with demonstrators exposed to a novel cage; (SINGLE) animals trained and tested without any social interaction; $\left({ }^{*}\right) P<0.05 ;\left(^{* *}\right) P<0.01 ;\left({ }^{* * *}\right) P<$ 
Table 3. Between-group comparisons of changes in the cumulative frequency distribution of response latencies recorded in the first and second sessions of the two-way avoidance training

\begin{tabular}{|c|c|c|c|c|c|}
\hline \multicolumn{3}{|c|}{ Session 1} & \multicolumn{3}{|c|}{ Session 2} \\
\hline Change & Dmax & $s$ & Change & Dmax & $s$ \\
\hline So > NSo & $0.1712^{\mathrm{a}}$ & 5.5 & So > NSo & $0.1310^{b}$ & 5.5 \\
\hline So > SINGLE & $0.2760^{\mathrm{a}}$ & 5.5 & So > SINGLE & $0.3251^{\mathrm{a}}$ & 5.5 \\
\hline NSo > SINGLE & $0.1116^{c}$ & 6.0 & NSo > SINGLE & $0.1941^{\mathrm{a}}$ & 5.5 \\
\hline
\end{tabular}

So $>$ NSo, for example, means that the proportion of responses emitted with latencies shorter than the point of Dmax was larger in observers from the So group than in observers from the NSo group. The point of Dmax showed the interval characterized by the largest deviation between two distributions that was subjected to a comparison. Size of samples compared for the first session: 394 for So, 400 for NSo, 392 for SINGLE; for the second session: 397 for So, 399 for NSo, and 394 for SINGLE.

The Kolmogorov-Smirnov two-sample test, ${ }^{\mathrm{a}} P<0.001,{ }^{\mathrm{b}} p<0.01,{ }^{\mathrm{c}} p<$ 0.05 .

To control the potential influence of aggression between the unfamiliar rats on the obtained results, the experimenter, who was blind to the experimental conditions, recorded the following aggressive behavioral episodes between demonstrators and observers: (1) threat posturing, (2) leaping, (3) boxing, (4) wrestling, and (5) biting. The only aggressive behavior that was observed was wrestling. However, there was no significant difference between groups (Sd: $1.9 \pm 0.3$; NSd: $1.5 \pm 0.3$ ). The results show that the improved learning is due to the socially transferred fear rather than aggression between rats.

\section{Interaction with a recently fear-conditioned demonstrator does not increase pain sensitivity or analgesia (Experiment 5)}

To control for the possible influence of different pain sensitivity thresholds in the observers paired with shocked demonstrators and the animals paired with the demonstrators that were merely exposed to the experimental cage, we carried out two acute pain tests (Fig. 4). The pain sensitivity of the observers was measured in the hot plate test $1 \mathrm{~d}$ before (baseline value), immediately after, and $1 \mathrm{~d}$ after the social interaction. There was no difference in pain sensitivity threshold between the So $(n=8)$ and NSo $(n=8)$ groups either immediately after or $1 \mathrm{~d}$ after the social interaction (as was confirmed by Wilcoxon matched pairs test). The only difference observed was between pre- and post-interaction periods $(P<0.05$; Wilcoxon matched pairs test). Pain sensitivity threshold was also assessed in the tail flick test three times before (baseline value), immediately after, and $1 \mathrm{~d}$ after the social interaction. Again, there was no difference in the pain sensitivity threshold between the So $(n=8)$ and NSo $(n=8)$ groups either immediately after or $1 \mathrm{~d}$ after the social interaction (as was confirmed by Wilcoxon matched pairs test). These results indicate that improved acquisition and retention of aversively motivated behaviors are not due to changes in pain sensitivity threshold.

\section{Discussion}

The main findings of the present study are the following:

1. During social interaction with a recently fear-conditioned partner, both observers and demonstrators exhibit social exploratory behaviors rather than aggressive behaviors.

2. Learning and memory in a shock-motivated shuttle avoidance task are facilitated in rats that underwent the social interaction with a partner that had been fear-conditioned.
3. A brief social interaction with a recently fear-conditioned partner immediately before fear conditioning improves conditioned freezing measured on the next day; this effect can be also seen in the rats that are unfamiliar to each other.

4. A brief social interaction with a recently fear-conditioned partner causes neither analgesia nor increase in pain sensitivity.

These data suggest that fear is transferred between conspecifics through social interaction and promotes both active and passive defensive responses in observers that interact with a recently shocked demonstrator.

One possibility to account for the social transfer of fear is that the social interaction between shocked demonstrators and observers consists of agonistic behavior, which is well known to increase emotional responding in a variety of threatening situations (Stam et al. 2000; Razzoli et al. 2007). To temper the influence of aggressive behavior in our model, we used familiar rats that were interacting in their home cages or, in the last experiment, unfamiliar rats that were extensively habituated to the cage where the interaction took place. This allowed us to avoid the resident-intruder situation, in which aggressive interaction is much more probable. Moreover, a careful analysis of behavior of both demonstrators and observers during the social interaction showed that neither observers nor demonstrators exhibited aggressive behaviors. This suggests that the augmented acquisition and retention of aversively motivated learning in rats exposed to a shocked demonstrator was not due to an agonistic encounter immediately prior to conditioning.

Demonstrators, especially those that were fear-conditioned, showed mainly nonsocial exploratory behaviors like rearing. On the other hand, observers were involved in considerably more social exploratory behaviors directed at their partners, such as sniffing in the anogenital area and allogrooming. "Allogrooming" is defined as a direct grooming of a partner's head and neck that

A.
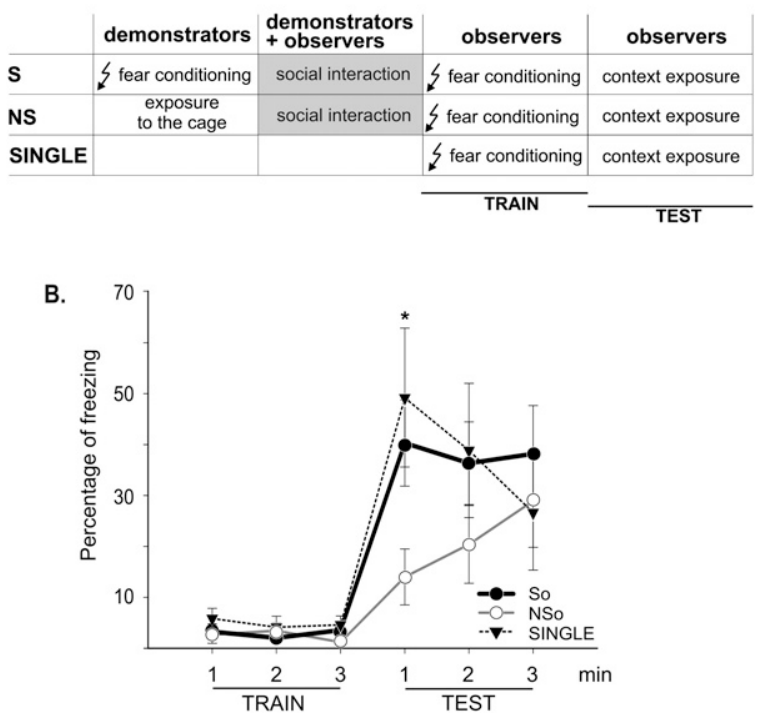

Figure 2. Interaction with a recently fear-conditioned partner improves fear memory. (A) Scheme of the experiment. (B) Familiar rats: Percentage of freezing measured during $3 \mathrm{~min}$ preceding a footshock during the training day (TRAIN) and 3 min during exposure to the experimental cage on the test day (TEST). (So) Observers paired with fear-conditioned demonstrators; (NSo) observers paired with demonstrators merely exposed to a novel cage; (SINGLE) animals trained and tested without any social interaction; $\left(^{*}\right) P<0.05$. 


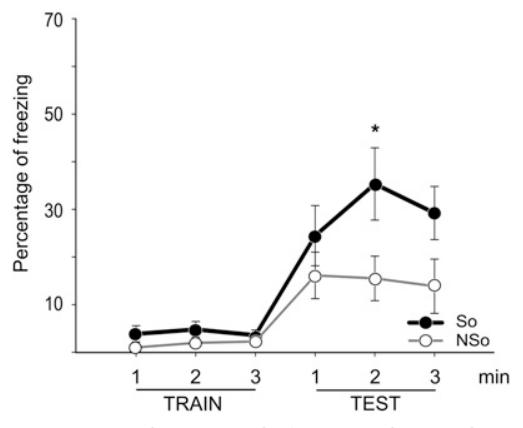

Figure 3. Interaction with a recently fear-conditioned partner improves fear memory also in unfamiliar rats. Percentage of freezing measured during 3 min preceding a footshock during the training day (TRAIN) and 3 min during exposure to the experimental cage on the test day (TEST). (So) Observers paired with fear-conditioned demonstrators; (NSo) observers paired with demonstrators merely exposed to a novel cage; $\left(^{*}\right) P<$ 0.05 .

has an investigative and affiliative meaning (Laviola et al. 2004; Barnett 2005). It may be an olfactory investigation of the sebum from the back of the animal. This behavior was much more intense in the observers paired with shocked demonstrators. Such results support the hypothesis that emotional arousal may be transferred between demonstrators and observers via alarm pheromones. Kiyokawa et al. (2004a, 2006) showed that the alarm pheromones excreted in the rat perianal region can change both autonomic responses (stress-induced hyperthermia) and increase defensive and risk assessment behaviors. In line with the hypothesis about pheromonal communication between our rats, it has been shown that chemosignals emitted by a recently fear-conditioned mouse can modify fear learning of another otherwise naïve mouse (Bredy and Barad 2008). In contrast to our results, however, Bredy and Barad (2008) found that exposing mice to a recently conditioned conspecific or a urinary chemosignal from shocked conspecifics impairs auditory fear conditioning. It is not entirely clear what accounts for this disparity, but it could involve differences between rats and mice, the nature of the conditioning in the demonstrators, and differences in behavioral paradigms (contextual vs. auditory fear conditioning). This issue certainly deserves further study.

It is possible that social interaction with a shocked conspecific affects endogenous analgesic mechanisms to influence shock-motivated learning. However, we did not observe any differences in responses to acute pain between the observers paired with either shocked or non-shocked demonstrators. In contrast, Fanselow (1985) has shown that odors released by stressed rats produce analgesia in unstressed conspecifics. The difference between Fanselow's results and ours may stem from the different procedures that were used. Fanselow measured persistent pain response (the stereotypical formalin-induced paw lick or paw lift), and we used the tests that measure acute pain responses. Moreover, Fanselow tested the observers in the cages where the donors previously obtained footshocks. In our experiments, the observers were tested in the experimental cage after the brief social interaction that took place in their home cage or in a novel cage, and the experimental cages were thoroughly cleaned between rats. Information transferred by freely moving demonstrator rats is likely to be different from that transferred by odors alone. One can also suppose that the observers' response strictly depends on the experimental context.

Another possibility is that social interaction influences locomotor behavior in the observers that affects learning by promoting either active coping strategies (avoidance responding) or passive coping strategies (defensive immobility). However, the data from Experiments 2-4 suggest that exposure to a shocked demonstrator facilitates the acquisition and retention of both active avoidance and conditioned immobility (freezing). This suggests that a change in locomotor activity per se in observers is not likely to account for their performance in aversively motivated learning tasks. Alternatively, our data suggest that the modulation of aversively motivated learning by social transfer of fear may involve either active or passive coping strategies depending on which one is best suited to the situations encountered by the observers. The social interaction might therefore transfer a fear state that fosters the acquisition and retention of defensive behaviors that are appropriate to any novel aversive situation.

It is also possible that animals that had a social interaction learn more slowly than noninteracting rats (SINGLE groups). Indeed, in the fear-conditioning paradigm, we observed relatively high levels of freezing in singly housed animals that did not have any social interaction. Their freezing was comparable with the level of freezing showed by the observers paired with fearconditioned demonstrators. However, comparison of learning efficacy in the two-way avoidance training showed that the animals that had a social interaction acquired the two-way avoidance response faster than the noninteracting animals. Especially the encounter with the fear-conditioned partner significantly improved learning. This result shows that a brief social interaction itself does not impede learning. Interestingly, single housing seems to promote defensive immobility rather than active defensive responses.

Pair-housing in the present experiments could lead to a phenomenon known as "social buffering." In this phenomenon, stress caused by aversive training might be mitigated by the presence of a conspecific animal. In the pair-exposure paradigm, it has been shown that social buffering depends on a partner's stress status, with non-shocked partners being more efficient than shocked partners (Kiyokawa et al. 2004b). Moreover, Kiyokawa et al. (2007) showed that pair-housing for $24 \mathrm{~h}$ with an unfamiliar rat following auditory fear conditioning resulted in a suppressed autonomic response in a fear-conditioned animal. However, they did not observe any differences in freezing response. In our experimental paradigm, we observed relatively high levels of freezing in singly housed animals that did not have any social interaction. Therefore, to exclude the potential influence of social buffering in our paradigm, we decided to repeat Experiment 3 with single-housed rats. The pattern of obtained results was, however, very similar to those obtained in pair-housed animals. Thus, we
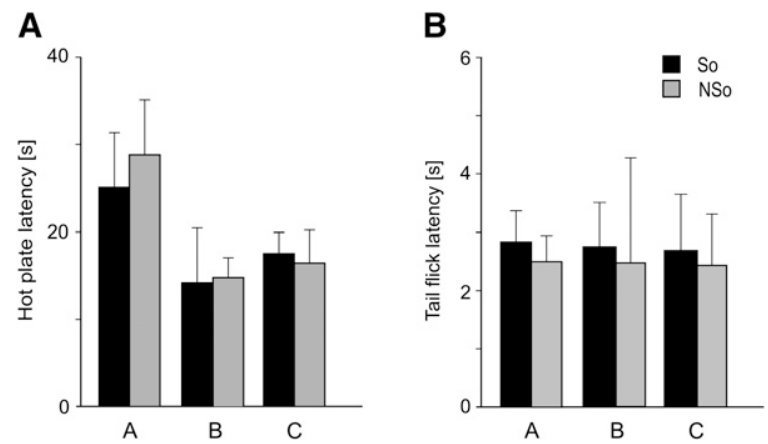

Figure 4. Interaction with recently fear-conditioned partner causes neither increase in pain sensitivity nor analgesia. Both hot plate $(A)$ and tail-flick $(B)$ tests showed no differences in response to acute pain between the observers paired with either shocked (So) or non-shocked (NSo) demonstrators. Pain sensitivity thresholds were measured $(A)$ before the social interaction (baseline level), (B) immediately after the social interaction, and (C) $24 \mathrm{~h}$ after the social interaction. 
can conclude that the effects of socially transferred fear can be observed irrespective of the type of housing.

Most of our experiments were carried out on familiar rats; however, Experiment 4, which was designed as a control of the social buffering effect, was conducted on unfamiliar conspecifics. It raises the problem of the role of familiarity of interacting animals. To date, there are only very limited data on this subject. Langford et al. (2006) observed modulation of pain sensitivity in mice produced by exposure to their cage mates in pain, but not to strangers. On the other hand, Saggerson and Honey (2006) found that familiarity with a demonstrator retarded the observational learning of another rat. We obtained very similar data for familiar and unfamiliar animals. It seems that the clear difference between our results and those of Langford et al. (2006) may stem from differences in the behavior of rats and mice in such a situation.

Empathy can be viewed as a phylogenetically continuous phenomenon, in its simplest forms operating as an emotional contagion. Collectively with our previous results (Knapska et al. 2006), the present data seem to be consistent with a perceptionaction model of empathy (Preston and deWaal 2002). The model predicts that the perception of an object's state (demonstrator in our model) activates the subject's (observer in our model) corresponding representations, which, in turn, activate somatic and autonomic responses. Such an organization of the nervous system, which probably is the precursor to empathy, should, in turn, facilitate appropriate responses to the environment. The increased arousal/vigilance that we have seen in the observers apparently helped them to adapt to the specific requirements of the experimental tasks. Moreover, it seems probable that emotional state transferred by a stressed demonstrator leads to increased excretion of arousal-released adrenal stress hormones that facilitate memory consolidation (McGaugh 2004).

Collectively, the obtained data suggest that a brief social interaction with a cage mate that had undergone an aversive learning experience promotes aversive learning in an otherwise naive animal. The effects observed in the present study were seen in all behavioral paradigms that we used; although the observed changes in animals' behavior were rather short, they were able to improve learning and memory. We hope that these models can be further used to study neuronal correlates of social interaction and communication.

\section{Materials and Methods}

\section{Experiment 1: Analysis of behavior during social interaction}

\section{Subjects}

Experimental subjects were 30 adult, experimentally naïve male Wistar rats (250-300 $\mathrm{g}$ at the beginning of the experiment), supplied by the Nencki Institute Animal House. After arrival, the animals were randomly paired, and for the following month, they were housed in pairs in standard home cages $(43.0 \times 25.0 \times 18.5 \mathrm{~cm})$ under a 14/10 light-dark cycle, with food and water provided ad libitum. The rats were habituated to the experimenter's hand, transport, and separation for $14 \mathrm{~d}$ preceding the experiment. The experiment was carried out in accordance with the Polish Act on Animal Welfare, after obtaining specific permission from the First Warsaw Ethical Committee on Animal Research.

\section{Behavioral apparatus}

The training was performed in a shuttle box $(62.0 \times 18.0 \times 29.0 \mathrm{~cm})$ (Zielinski and Nikolaev 1997) that consisted of two identical opaque dark acrylic compartments separated by a wall with a rectangular $(6.5 \mathrm{~cm}$ wide, $7 \mathrm{~cm}$ high) opening with a sill situated on the gridfloor level. The cage was covered by a movable transparent acrylic ceiling and illuminated by two 5-W lamps mounted centrally just below the ceiling, one over each compartment. On each wall opposite to a central partition, a 10-cm loudspeaker was mounted outside of the apparatus and $15 \mathrm{~cm}$ above the floor. The loudspeakers delivered white noise, $70 \mathrm{~dB}$. Crossing through the opening was recorded by light photocells mounted $4 \mathrm{~cm}$ to either side of the central partition, $5 \mathrm{~cm}$ above the floor level. The floor was constructed from 32 stainless steel bars, $0.4 \mathrm{~cm}$ in diameter, and located parallel to the central partition $1.4 \mathrm{~cm}$ apart from each other. The shuttle-box apparatus was placed in a dark, sound-attenuating room. Subjects' behavior was observed on a television monitor in an adjoining room in which equipment for automatic programming of the experiment and recording of data was also located. For fear conditioning, the central partition was removed.

\section{Procedure}

\section{Group assignment}

The animals were randomly divided into S (Shocked) and NS (NonShocked) groups. Then, in each cage, one subject was marked as a demonstrator (d) and the other as an observer (o). In the $\mathrm{S}$ group, the demonstrators (Sd) were taken from their home cages for aversive conditioning (Pavlovian contextual fear conditioning). When the demonstrators were trained, their cohabitants (observers, So) were kept in the home cages in a different sound-attenuating room and were not able to hear the vocalization of demonstrators. Immediately after the training, the demonstrators were placed back in their home cages and allowed to interact with the observers for $10 \mathrm{~min}$. The NS group, composed of rats treated like those from the S group, except that the NS demonstrators (NSd) were exposed to the experimental cage without any training.

\section{Contextual fear conditioning}

The demonstrators $(\mathrm{Sd})$ were subjected to the Pavlovian contextual fear conditioning. The training consisted of a 2-min adaptation period and 9 footshocks lasting $1 \mathrm{sec}$, of $1.3-\mathrm{mA}$ intensity, which were applied with interstimulus intervals of $55 \mathrm{sec}$. The animals were removed from the experimental cage $1 \mathrm{~min}$ after the last footshock was applied.

\section{Analysis of behavior during social interaction}

All animals were habituated for $3 \mathrm{~d}$ (one 20-min session per day) to the experimental room, separation, and marking process. Each animal was marked with two different color spots (one on the head and one on the central part of the back). On the experimental day, the demonstrators from the $S$ group were subjected to Pavlovian contextual fear conditioning and then returned to the home cages, where they interacted with the observers. The 10-min social interaction was video recorded, and the behavior of observers and demonstrators was analyzed with the use of BehaView software (http://www.pmbogusz.net/software/). The experimenter, who was blind to the experimental conditions, recorded the number and/or duration of the following behavioral episodes: (1) rearing, (2) self-grooming, (3) sniffing partner in the anogenital area, (4) sniffing partner in the head area, (5) allogrooming (grooming of the partner), (6) threat posturing, (7) leaping, (8) boxing, (9) wrestling, and (10) biting.

\section{Experiment 2: Learning of two-way avoidance after social interaction with a partner that was fear-conditioned}

Experimental subjects were 40 adult, experimentally naive male Wistar rats (250-300 $\mathrm{g}$ at the beginning of the experiment) obtained and housed as described in Experiment 1. In the case of the SINGLE group, the rats were single-housed in standard home cages $(43.0 \times 21.0 \times 20.5 \mathrm{~cm})$. The behavioral apparatus and procedure were the same as in the first experiment, except that in Experiment 2, the animals were not marked with color spots, and the observers were trained in two-way avoidance immediately after social interaction and $24 \mathrm{~h}$ later. 


\section{Avoidance training}

The observers in Experiment 3 (So, NSo) and the rats from the SINGLE group were subjected to two-way avoidance training. At the beginning of the training session, a rat was placed in the left compartment of the shuttle box, close to and facing the end wall. After $20 \mathrm{sec}$ of adaptation, a trial started with 70-dB white noise (conditioned stimulus, CS) onset, and, $5 \mathrm{sec}$ later, the CS was accompanied by the scrambled footshock of $1.0-\mathrm{mA}$ intensity (unconditioned stimulus, US). Moving to the opposite compartment within the first $5 \mathrm{sec}$ of CS precluded the footshock, immediately terminated the CS, and was scored as an avoidance response. Similar response but after the US onset immediately coterminated the CS and US and was scored as an escape response. The maximal shock duration was $30 \mathrm{sec}$. Thus, in case of no response during that period, a trial was automatically terminated. The intertrial intervals (ITI) lasted 14,20, or $26 \mathrm{sec}$ and varied in a semi-random order. During ITIs, subjects were permitted to move in any direction, so they could cross away from or back into the compartment in which they had been previously. The next trial always started in the compartment in which the subject was actually present. Crossings between compartments when neither CS nor US was present were counted during the period preceding the first trial (adaptation period, a) and in the subsequent ITIs (intertrial responses, ITRs). The animals were removed from the experimental chamber within $30 \mathrm{sec}$ after the last trial. The latencies of instrumental responses and numbers of avoidance responses and crossings were recorded. Since the intertrial periods varied in length, the frequency of crossings (the number of reactions per minute) was calculated, dividing the number of crossings by the length of the corresponding part of the procedure, that is, $20 \mathrm{sec}$ for the adaptation period and 1006 sec $(16.77 \mathrm{~min})$ for the sum of the intertrial periods. The training consisted of two 50-trial sessions that took place on two consecutive days.

\section{Experiment 3: Effects of socially transferred fear on fear memory of an observer rat}

\section{Subjects}

The subjects were 58 experimentally naïve male Long-Evans rats (200-224 g) obtained from a commercial supplier (Harlan SpragueDawley). After arrival, the animals were randomly paired and housed in pairs in standard plastic cages $(43.0 \times 21.0 \times 20.5 \mathrm{~cm})$ for the following $2 \mathrm{wk}$. Additionally, in the case of one control group (SINGLE), the rats were single-housed in standard home cages $(43.0 \times 21.0 \times 20.5 \mathrm{~cm})$. All animals were kept under a 14/10 light-dark cycle, with food and water provided ad libitum. The rats were habituated to the experimenter's hand (for $13 \mathrm{~d}$ ), as well as to transport and separation for $5 \mathrm{~d}$ preceding the experiment. All experiments were carried out in accordance with guidelines approved by the University of Michigan University Committee on Use and Care of Animals.

\section{Behavioral apparatus}

Eight identical observation chambers $(30.0 \times 24.0 \times 21.0 \mathrm{~cm}$; Med-Associates) were used for all phases of training and testing. The chambers were constructed from aluminum (two side walls) and Plexiglas (rear wall, ceiling, and hinged front door) and were situated in sound-attenuating chests located in an isolated room. The floor of each chamber consisted of 19 stainless-steel rods $(4 \mathrm{~mm}$ diameter) spaced $1.5 \mathrm{~cm}$ apart. The rods were wired to a shock source and solid-state grid scrambler (Med-Associates) for delivery of the footshock (US). Background noise $(65 \mathrm{~dB})$ was provided by ventilation fans built into the chests, and house lights within the chambers and lights within the room provided illumination. Between tests, the chambers were washed with hot water with a cleanser, wiped dry with a paper towel, and spread with $1 \%$ acetic acid solution. Stainless steel pans containing a thin film of $1 \%$ acetic acid solution were placed underneath the grid floors before the animals were placed inside the boxes. Each conditioning chamber rested on a load cell platform that was used to record chamber displacement in response to each rat's motor activity. To ensure interchamber reliability, each load cell amplifier was calibrated to a fixed chamber displacement. The output of the load cell of each chamber was set to a gain that was optimized for detecting freezing behavior. Load cell amplifier output from each chamber was digitized and acquired on-line using Threshold Activity software (Med-Associates).

\section{Procedure}

The rats were transported to the laboratory in their home cages. On the conditioning day (TRAIN), the demonstrators were placed in the conditioning chambers. They received Pavlovian contextual fear conditioning that consisted of 10-footshock $(1.0 \mathrm{~mA}$, $1 \mathrm{sec}$ ) presentations with a 60 -sec intertrial interval. The training began $3 \mathrm{~min}$ after placing an animal in the chamber and ended $1 \mathrm{~min}$ after the shock. Then demonstrators returned to their home cages and were allowed to interact with observers for $10 \mathrm{~min}$. Afterward, the observers were subjected to contextual fear conditioning (3-min adaptation period and one footshock, $1.0 \mathrm{~mA}$, $1 \mathrm{sec})$. The training ended 1 min after the shock. In the NS group, the demonstrators were merely exposed to the experimental cage without any training for the same amount of time as the animals from the Sd group. Additionally, the rats that were single-housed and did not interact with another rat were trained in the same behavioral paradigm (SINGLE group). Twenty-four hours after training, contextual fear of observers and the rats from the SINGLE group was assessed by returning the rats to the conditioning chambers and measuring freezing behavior during a 3-min period.

\section{Experiment 4: Effects of fear transferred by recently fear-conditioned unfamiliar demonstrator on fear memory of observer}

The subjects were 48 experimentally naïve male Long-Evans rats (200-224 g) obtained from a commercial supplier (Harlan Sprague-Dawley). They were individually housed in transparent plastic cages hanging from a standard stainless-steel rack. All animals were kept under a 14/10 light-dark cycle, with food and water provided ad libitum. The rats were habituated to the experimenter's hand (for $13 \mathrm{~d}$ ), as well as for transport and separation for $5 \mathrm{~d}$ preceding the experiment. Additionally, the rats were habituated for $5 \mathrm{~d}$ preceding the experiment to "interaction cages" that were transparent standard plastic cages $(43.0 \times 21.0 \times$ $20.5 \mathrm{~cm})$. The rats were transported to the laboratory in the transport cages. The experimental procedure was as described in Experiment 3.

\section{Experiment 5: Hot plate and tail-flick tests}

Experimental subjects were 64 adult, experimentally naïve male Wistar rats (250-300 g at the beginning of the experiment) obtained and housed as described in Experiment 1 . The procedure was the same as in the first experiment, except that in Experiment 5 , the animals were not marked with color spots, and $1 \mathrm{~d}$ before, immediately after and $1 \mathrm{~d}$ after the social interaction, the pain sensitivity of the observers was measured in the hot plate test (32 rats) or $3 \mathrm{~d}$ before, immediately after and $1 \mathrm{~d}$ after the social interaction in the tail-flick test (32 rats).

\section{Fear-conditioning cage}

A chamber $(30.0 \times 24.0 \times 21.0 \mathrm{~cm}$; Med-Associates $)$ that was used for fear conditioning of the demonstrators was constructed from aluminum (two side walls) and Plexiglas (rear wall, ceiling and hinged front door) and was situated in a sound-attenuating chest located in an isolated room. The floor of the chamber consisted of 19 stainless-steel rods ( $4 \mathrm{~mm}$ diameter) spaced $1.5 \mathrm{~cm}$ apart. The rods were wired to a shock source and solid-state grid scrambler (Med-Associates) for delivery of the footshock (US). Background noise $(65 \mathrm{~dB})$ was provided by a ventilation fan built into the chest, and house light within the chamber and lights within the room provided illumination. Between tests, the chamber was washed with water with a cleanser, wiped dry with a paper towel, and then wiped with a $1 \%$ ethanol solution. Stainless steel pans 
were placed underneath the grid floor before the animals were placed inside the box. The freezing response was recorded by the camera placed in front of the cage and the computer system located in the adjoining room.

\section{Hot plate test}

In the hot plate test, rats were placed on a Cooper plate heated by water of $56^{\circ} \mathrm{C}$, and the latency to lick a hind paw was recorded. The plate $(30.0 \times 30.0 \mathrm{~cm})$ was enclosed by a $42.0-\mathrm{cm}-\mathrm{high}$ transparent plastic box. The maximum trial length permitted was $60 \mathrm{sec}$.

\section{Tail-flick test}

The tail-flick test was performed using the Letica S.A LI 7106 light beam analgesimeter. The heat produced by a halogen light $(12 \mathrm{~V}$ DC, $100 \mathrm{~W}$ ) placed in a moveable lamp was concentrated on the dorsal surface of the rat's tail. The animal was restrained by the experimenter's hand to leave its tail completely free, but in such a way that it remained lying between the two adjustable sections of the experimental unit base. The latencies to flick the tail in response to heat were measured in three trials with maximum permitted time of $7 \mathrm{sec}$, separated by 3 -min periods of rest in a small plastic cage. The mean of the three measurements was used in further analysis for the sessions that took place immediately after and $1 \mathrm{~d}$ after the social interaction. For the baseline response, the mean of the nine measurements was calculated.

\section{Acknowledgments}

This work was partly supported by the Polish Ministry of Science and Higher Education (grant NN303 088834 to T.W.); E.K. acknowledges support from the Foundation for Polish Science through its programme KOLUMB. We thank Pawel Boguszewski for providing us with the BehaView software, as well as Kaitlyn Matz and Joanna Sadowska for excellent technical assistance.

\section{References}

Barnett SA. 2005. Ecology. In The behavior of the laboratory rat (ed. IQ Whishaw, B Kolb), pp. 15-24. Oxford University Press, New York.

Bredy TW, Barad M. 2008. Social modulation of associative fear learning by pheromone communication. Learn Mem 30: 12-18.

Church RM. 1959. Emotional reactions of rats to the pain of others. J Comp Physiol Psychol 52: 132-134.

Decety J, Lamm C. 2006. Human empathy through the lens of social neuroscience. Scientific World Journal 6: 1146-1163. de Waal FB. 2008. Putting the altruism back into altruism: The evolution of empathy. Annu Rev Psychol 59: 279-300.

Fanselow MS. 1985. Odors released by stressed rats produce opioid analgesia in unstressed rats. Behav Neurosci 99: 589-592.

Hatfield E, Cacioppe JT, Rapson RL. 1994. Emotional contagion. Cambridge University Press, New York.

Kiyokawa Y, Kikusui T, Takeuchi Y, Mori Y. 2004a. Alarm pheromones with different functions are released from different regions of the body surface of male rats. Chem Senses 29: 35-40.

Kiyokawa Y, Kikusui T, Takeuchi Y, Mori Y. 2004b. Partner's stress status influences social buffering effects in rats. Behav Neurosci 118: 798-804.

Kiyokawa Y, Shimozuru M, Kikusui T, Takeuchi Y, Mori Y. 2006. Alarm pheromone increases defensive and risk assessment behaviors in male rats. Physiol Behav 87: 383-387.

Kiyokawa Y, Takeuchi Y, Mori Y. 2007. Two types of social buffering differentially mitigate conditioned fear responses. Eur J Neurosci 26: 3606-3613.

Knapska E, Nikolaev E, Boguszewski P, Walasek G, Blaszczyk J, Kaczmarek L, Werka T. 2006. Between-subject transfer of emotional information evokes specific pattern of amygdala activation. Proc Natl Acad Sci 103: 3858-3862.

Langford DJ, Crager SE, Shehzad Z, Smith SB, Sotocinal SG, Levenstadt JS, Chanda ML, Levitin DJ, Mogil JS. 2006. Social modulation of pain as evidence for empathy in mice. Science 312: 1967-1970.

Laviola G, Adriani W, Rea M, Aloe L, Alleva E. 2004. Social withdrawal, neophobia, and stereotyped behavior in developing rats exposed to neonatal asphyxia. Psychopharmacology 175: 196-205.

Maren S. 2001. Neurobiology of Pavlovian fear conditioning. Annu Rev Neurosci 24: 897-931.

McGaugh JL. 2004. The amygdala modulates the consolidation of memories of emotionally arousing experiences. Annu Rev Neurosci 27: $1-28$.

Olsson A, Phelps EA. 2007. Social learning of fear. Nat Neurosci 10: 10951102.

Preston SD, de Waal FB. 2002. Empathy: Its ultimate and proximate bases. Behav Brain Sci 25: 1-20.

Razzoli M, Carboni L, Guidi A, Gerrard P, Arban R. 2007. Social defeatinduced contextual conditioning differentially imprints behavioral and adrenal reactivity: A time-course study in the rat. Physiol Behav 92: 734-740.

Saggerson AL, Honey RC. 2006. Observational learning of instrumental discriminations in the rat: The role of demonstrator type. QJExp Psychol (Colchester) 59: 1909-1920.

Stam R, Bruijnzeel AW, Wiegant VM. 2000. Long-lasting stress sensitization. Eur I Pharmacol 405: 217-224.

Zielinski K, Nikolaev E. 1997. Changes of intertrial response rate with elapse of time after two-way avoidance trial in rats. Acta Neurobiol Exp (Warsz) 57: 41-47.

Received October 14, 2009; accepted in revised form November 11, 2009. 


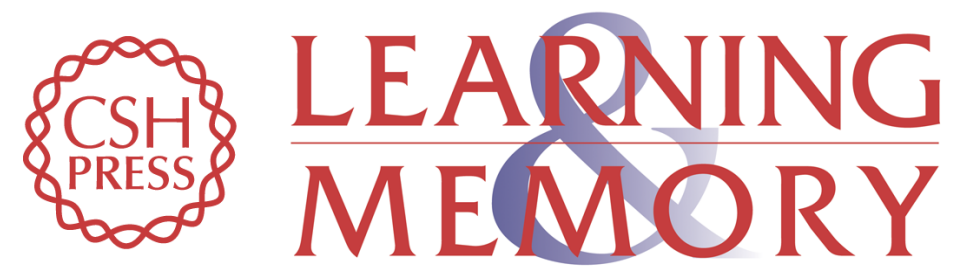

\section{Social modulation of learning in rats}

Ewelina Knapska, Marta Mikosz, Tomasz Werka, et al.

Learn. Mem. 2010, 17:

Access the most recent version at doi:10.1101//m.1670910

References This article cites 20 articles, 2 of which can be accessed free at: http://learnmem.cshlp.org/content/17/1/35.full.html\#ref-list-1

License

Email Alerting Receive free email alerts when new articles cite this article - sign up in the box at the Service top right corner of the article or click here. 